\title{
Panorama das pesquisas em educação a distância no Brasil
}

\author{
Gardênia da Silva Abbad \\ Universidade de Brasilia \\ Thaís Zerbini \\ Universidade de São Paulo - Ribeirão Preto \\ Daniela Borges Lima de Souza \\ Universidade de Brasília
}

\begin{abstract}
Resumo
O investimento em treinamento, desenvolvimento e educação continua sendo um diferencial competitivo para as organizações. Nesse contexto, a educação a distância (EAD) é analisada como uma possibilidade viável na construção de mecanismos que favoreçam a aprendizagem e qualificação contínuas e ao longo da vida. O presente estudo se insere nesse conjunto de reflexões. Seu objetivo é descrever brevemente o contexto e o foco das pesquisas sobre treinamentos a distância (TAD) no Brasil, no período de 2003 a 2009, a partir de pesquisa bibliográfica e de dados do Anuário Brasileiro Estatístico de Educação Aberta e a Distância (ABRAED). A revisão da produção de conhecimentos em EAD no Brasil mostra a necessidade de mais pesquisas nas áreas de educação corporativa, qualificação profissional e avaliação. O ritmo das pesquisas é incompatível com o acelerado crescimento desse tipo de aprendizagem em organizações. Outras considerações são realizadas e uma agenda de pesquisa é proposta.

Palavras-chave: educação a distância; treinamento a distância; treinamento e desenvolvimento.
\end{abstract}

\begin{abstract}
Overview of researches in distance education in Brazil. Investment in training, development and education is a competitive advantage for organizations. In this context, distance learning education (EAD) is regarded as a viable possibility in the construction of mechanisms which encourage continuous learning and acquirement of skills throughout life. The present study is within the set of reflections. Its goal is to briefly describe the context and focus of research on distance training in Brazil, from 2003 to 2009, based on literature and data review at the Brazilian Statistical Yearbook of Education Open and Distance (ABRAED). The production review of knowledge in distance education in Brazil shows the need for more research in corporate education, professional training, and evaluation. The pace of research is incompatible with the rapid growth of this type of learning in organizations. Other considerations are taken and a research agenda is proposed.
\end{abstract}

Keywords: e-learning; blended learning; training and development.

A $\mathrm{s}$ intensas e rápidas mudanças econômicas e sociais que perpassam o mundo do trabalho têm exigido dos diversos atores desse cenário o investimento maciço em processos contínuos de aprendizagem que possibilitem a qualificação constante. Fazem parte dessas mudanças e solicitam aprendizagem contínua, o desenvolvimento constante de tecnologias e procedimentos, o surgimento de novos requisitos para trabalhadores e organizações, bem como a alteração no contexto e no conceito de organização (Sonnentag, Niessen, \& Ohly, 2004).

$\mathrm{O}$ investimento em eventos de treinamento, desenvolvimento e educação (TD\&E) é um dos diferenciais competitivos das empresas e precisa apresentar conexão direta com objetivos e com as metas da organização, bem como ser planejado e executado com o objetivo fundamental de evitar a obsolescência das organizações e promover inovações constantes, a partir do reconhecimento de necessidades impostas pelo cenário globalizado (Coelho Jr. \& Borges-Andrade, 2008).

Nesse contexto, a educação a distância (EAD) é analisada como uma possibilidade viável na construção de mecanismos que favoreçam a aprendizagem e a qualificação contínuas ao longo da vida, bem como apresenta, enquanto modalidade de ensino, condições de ensino adequadas para adultos. Além disso, por ser mediada por tecnologias da informação e comunicação (TICs), é plenamente utilizável no ambiente corporativo, já que possibilita o desenho de eventos educacionais focalizados em situações específicas, além de ampliar e democratizar o acesso às oportunidades de treinamento.

$\mathrm{O}$ presente artigo se insere nesse conjunto de reflexões e objetiva descrever brevemente o contexto e o foco das pesquisas 
sobre treinamentos a distância (TAD) no Brasil, no período de 2003 a 2009, a partir de pesquisa bibliográfica e de dados do Anuário Brasileiro Estatístico de Educação Aberta e a Distância (Instituto Monitor, 2007, 2008). A maior concentração de publicações sobre o tema no Brasil ocorreu no referido período. Analisam-se também, os principais conceitos que norteiam a condução de estudos nas diversas linhas de pesquisas nessa área, bem como a produção de conhecimentos sobre ensino EAD e TAD, de modo a identificar lacunas e descrever alguns resultados de pesquisas. Foram analisados artigos publicados em revistas de psicologia, administração, computação e educação, revisões de literatura sobre TD\&E e sobre e-learning. Os artigos foram localizados em bibliotecas virtuais e selecionados aqueles publicados em revistas científicas que adotam avaliação dos manuscritos por pares. $\mathrm{Na}$ amostra final predominaram artigos publicados em revistas de educação e psicologia. Foram analisadas também dissertações e teses. Foram consultados ainda, anuários estatísticos de 2007 e 2008 sobre educação aberta e a distância no Brasil.

As análises dos artigos são apresentadas nas seções seguintes, a saber: Conceitos norteadores das pesquisas em EAD e TAD; Resultados de pesquisas e lacunas na área; e Considerações finais.

\section{Conceitos norteadores das pesquisas em EAD e TAD}

A EAD, no mundo inteiro, é uma modalidade voltada à aprendizagem de adultos. Essa modalidade está vinculada a vários princípios educacionais, entre os quais o de aprendizagem aberta, aprendizagem ao longo de toda vida ou educação permanente. No Brasil, a EAD está sendo adotada na educação, em programas de qualificação e formação profissional, e em educação corporativa (Abbad, 2007).

A educação corporativa está crescendo rapidamente, calcada na oferta de cursos através de ambientes virtuais de aprendizagem que possibilitam ao estudante e ao professor a interação assíncrona, a qual permite aos participantes interagirem a qualquer momento, independente da sincronia de ações, e a veiculação de objetos de aprendizagem pela internet (Abbad, Carvalho, \& Zerbini, 2006). O apoio de novas tecnologias da comunicação e informação possibilitou a adoção de e-learning e blended learning em grande escala pelas universidades corporativas e pelas instituições de ensino.

$\mathrm{O}$ e-learning é a aprendizagem apoiada por recursos da web, que combina múltiplas mídias e tecnologias como: comunicação por satélite, vídeo, áudio, tecnologia multimídia, entre outras. O método de aprendizagem a distância tem sido aplicado de modo síncrono e assíncrono com a disponibilização de materiais para todos os participantes, os quais geralmente estão separados geograficamente uns dos outros. Blended learning é uma modalidade que combina a aprendizagem face a face com a aprendizagem mediada pela internet. Os cursos híbridos são aqueles em que uma parte significativa das atividades de ensino-aprendizagem foi transferida para ambientes online. Por isso, adoção do blended learning requer o redesenho de cursos e a redução do tempo gasto em sala de aula. Tais cursos não eliminam completamente a sala de aula, apenas reduzem bastante a sua ocorrência.
A oferta de cursos online é viabilizada por Learning Management Systems, conhecido como LMS, ou Sistemas de Gerenciamento de Cursos (SGC). Esses softwares são desenvolvidos de acordo com princípios didático-pedagógicos para auxiliar a promoção de ensino e da aprendizagem virtual. Possibilitam o armazenamento de grandes quantidades de informações e objetos de aprendizagem, bem como a criação de salas de aula virtuais, nas quais ocorrem interações síncronas (chats, aulas virtuais, videoconferências) e assíncronas (fóruns de discussão, wikis, e-mails) entre alunos, professores e demais participantes.

Escolas de Governo de diversos países como França, Espanha, Canadá e Brasil estão adotando a educação a distância, em todas as suas formas, na oferta de cursos para servidores públicos e para a comunidade. De acordo com Burguess e Russel (2003), muitas organizações após a tragédia do onze de setembro nos Estados Unidos da América (EUA), cancelaram viagens para treinamento e intensificaram a utilização de aprendizagem a distância. Os treinamentos têm sido oferecidos a milhares de pessoas simultaneamente, através de diversas tecnologias, entre as quais a transmissão via satélite. Pesquisas analisadas pelos autores supracitados mostraram que aproximadamente $24 \%$ das companhias estadunidenses estavam destinando recursos financeiros especialmente para programas de treinamento a distância.

Ainda que esta temática esteja em ebulição e necessite ser amplamente debatida, o que se pode perceber pela análise da literatura da área é que ainda existem consideráveis lacunas na produção de conhecimentos no contexto brasileiro e, por isso, analisar a literatura sobre e-learning e blended-learning se faz necessário e oportuno (Abbad, Carvalho, \& Zerbini, 2006).

\section{Resultados de pesquisas e lacunas na área}

Foram selecionadas, aproximadamente, trinta referências, entre artigos, dissertações de mestrado e teses de doutorado. Tais estudos foram analisados de acordo com determinadas categorias de conteúdo, a saber: (a) perfil dos participantes ou público-alvo dos cursos; (b) características dos conteúdos da aprendizagem; (c) situações e recursos de ensino-aprendizagem; (d) interações e interatividade; (e) suporte institucional e organizacional à aprendizagem a distância; (f) evasão em cursos a distância; e (g) avaliação da efetividade do e-learning ou blended learning. $\mathrm{Na}$ seção 2.1, discutem-se as cinco primeiras categorias de conteúdo. Na seção seguinte, são descritos os resultados da análise de artigos sobre evasão e, na seção 2.3, apresenta-se a análise da produção de conhecimentos sobre avaliação de cursos a distância.

\section{Resultados gerais da análise de sobre treinamento e educação a distância}

Quanto à faixa etária dos participantes dessas atividades, observa-se uma grande variedade de perfis de alunos de cursos a distância. Em cursos de graduação predominam os jovens, enquanto que em cursos de especialização e pós-graduação, nota-se uma participação maior de estudantes mais idosos e uma variação maior de idade entre os estudantes. Em organizações, os treinamentos a distância são oferecidos para clientelas internas 
(os empregados) e externas (clientes, fornecedores, parceiros, entre outros stakeholders).

Em cursos abertos destinados a formação profissional de curta duração há grande variação de idade, desde jovens de 16 a 18 anos até adultos com 65 anos (Carvalho, 2003; Zerbini, 2007). No Brasil, em escolas de governo, empresas estatais, órgãos públicos e no Sistema $\mathrm{S}$, as 41 unidades educacionais pesquisadas pelo Instituto Monitor (2008) oferecem cursos a distância a um público-alvo geograficamente disperso, pertencente a uma ampla faixa etária (18 a 60 anos), com um predomínio de adultos mais idosos (de 30 a 60 anos de idade), com nível superior de instrução, sendo que algumas escolas também oferecem cursos a alunos de nível de escolaridade médio e fundamental. Quanto ao nível hierárquico atendido por curso a distância, em ambientes corporativos no Brasil, um dos mais atendidos é o operacional (Instituto Monitor, 2007, 2008). Os treinamentos a distância oferecidos pelas organizações tem duração média de 41 horas, com um mínimo de 8 horas e um máximo de 255 horas.

Quanto às características do conteúdo da aprendizagem, observa-se que em organizações de trabalho, são ofertados cursos com uma ampla variedade de conteúdos relacionados a competências gerenciais, técnicas e instrumentais. Na educação corporativa brasileira é observada uma tendência de oferta de cursos de informática, gestão, educação e cidadania (Instituto Monitor, 2007, 2008). A duração dos cursos é bastante variada. Nas pesquisas estrangeiras e brasileiras, observam-se cursos de curta duração de oito horas (Coelho Jr., 2004; Nogueira, 2006) e de longa duração como cursos de graduação a distância (Parker, 1995, 1999; Xenos, Pierrakeas, \& Pintelas, 2002). Os conteúdos dos cursos são classificados principalmente nos domínios cognitivo e afetivo.

Quanto às situações e recursos de ensino-aprendizagem, observa-se que os treinamentos classificados como e-learning e o blended-learning adotam uma ampla variedade de procedimentos, recursos e meios instrucionais, calcados em recursos de multimídia com figuras e animações e recursos de áudio.

Quanto às interações entre pessoas (tutor-estudante, estudante-estudante, tutor-material, estudante-material), encontrou-se um aparente predomínio de situações de interações assíncronas entre os participantes e entre eles e os materiais. $\mathrm{O}$ e-learning e o blended learning incluem interações síncronas e assíncronas. Em cursos mistos, há também encontros face a face entre professores e alunos. Além disto, todos os materiais utilizados em situações de interação síncrona em tempo real podem ser digitalizados e disponibilizados como objetos de aprendizagem para estudo assíncrono. Observa-se a adoção (ou a sugestão) de comunicações bidirecionais escritas e faladas entre professor-aluno, aluno-aluno e aluno material com feedbacks personalizados, contingentes ao desempenho do aluno.

Um fato que merece ser mencionado é que o estudante adulto possui muitos papéis e responsabilidades concorrentes com as atividades de estudo. Isto, em alguns casos, inviabiliza a participação do aluno em encontros síncronos durante o dia. No Brasil, observou-se que alunos de cursos a distância, principalmente em treinamentos corporativos não obrigatórios e em cursos técnicos abertos ao público, costumam acessar os websites dos cursos à noite (das 18 à meia noite) e nos finais de semana (De Paula e Silva, 2004; Zerbini, 2003).

Interatividade, compreendida como o grau de interação entre o aluno e o material do curso, é maior em e-learning autoinstrucional, pois os materiais e recursos precisam incorporar as funções didáticas do professor e garantir feedbacks informativos e contingentes ao desempenho do aluno no treinamento. Nas pesquisas são citados problemas associados ao uso dos recursos de interação no e-learning. Os websites estão sendo usados como grandes livrarias virtuais e com pouca interação entre os participantes dos cursos, alunos e professores. O estudo feito por Shemla e Nachmias (2007) na Universidade de Tel Aviv confirma essa informação.

Quanto ao uso dos websites para apresentar materiais não textuais como meios de representação dos conteúdos (instrucionais, não decorativos), observa-se uso pouco frequente de imagens, simulações, gráficos, desenhos ou esboços, vídeos e som. O uso de vídeos com demonstrações apóia a retenção de conhecimentos e aumenta a motivação para aprender e facilita a aprendizagem. O uso de vídeos para ensinar habilidades clínicas a estudantes de enfermagem para apoiar as demonstrações de procedimentos pelo professor foi relatado e avaliado positivamente por Kelly, Lyng, McGrath e Cannon (2009). A pesquisa dos autores é um exemplo do uso efetivo de recursos visuais para facilitar a aprendizagem. Chama a atenção também o survey realizado por Gormley, Collins, Boohan, Bickle e Stevenson (2009) sobre a adoção do e-learning no ensino de habilidades clínicas a estudantes de medicina.

Quanto às interações, observou-se, ainda, que a interação mais frequente ocorre entre o estudante e os materiais instrucionais, disponibilizados nos Ambientes Virtuais de Aprendizagem (AVAs). Interações entre o estudante e o professor e estudante-estudante são mais utilizadas em cursos de média e longa-duração a distância. São mais raras as interações entre estudantes para estudo dos materiais e realização de exercícios. Os resultados de dois estudos nacionais mostraram que a busca espontânea de ajuda do tutor pelo aluno é pouco frequente (Abbad, Carvalho, \& Zerbini, 2006; De Paula e Silva, 2004).

Os recursos de interação síncrona, ao que parece, beneficiam um número relativamente reduzido de alunos. A participação de todos os alunos em grupos de discussão não é tarefa fácil, pois exige a participação de um moderador que organiza a atividade, acompanha e oferece feedbacks aos alunos. Há alunos que não participam espontaneamente de discussões assíncronas. A participação de alunos em chats bem planejados parece motivar as discussões e as interações aluno-aluno e professor-aluno. Porém, na amostra de artigos pesquisados, não foram encontradas pesquisas sobre interações desse tipo.

Segundo Abbad, Carvalho e Zerbini (2006), há poucas pesquisas investigando o efeito exercido por recursos e estratégias nas reações, na aprendizagem e na transferência de aprendizagem para o trabalho. Quanto a variáveis de contexto de estudo do aluno de curso a distância, foram encontrados poucos trabalhos sobre a importância do suporte da organização ao e-learning, na educação corporativa, e do suporte institucional em cursos a distância em ambientes universitários. Os resultados da pesquisa de Almeida (2007) indicam que a falta de suporte 
da organização ao estudo pode levar à evasão. Em outro estudo, de Coelho Jr., Abbad e Vasconcelos (2008), a falta de suporte à aprendizagem esteve relacionada negativamente com transferência de um treinamento online.

O estudo propiciado pela EAD e, em especial, pelo e-learning impõe vários desafios aos pesquisadores desta área. Variáveis de diferentes contextos de estudo do aluno passam a afetar a participação e o rendimento em cursos a distância, o que, consequentemente, deveria resultar em mais pesquisas. Há restrições situacionais do ambiente de trabalho, como: tarefas incompatíveis com o estudo, ruídos, interferências e interrupções, pressões de tempo, conflito de papéis, falta de apoio de chefes a empregados e servidores públicos em treinamentos corporativos. Além desses desafios, o estudante de e-learning, tanto em ambiente organizacional como em acadêmico, está submetido a fatores presentes em outros contextos como o familiar e em outros locais nos quais ele acessa os materiais de estudo. Pouco se sabe sobre a influência de variáveis desse tipo no comportamento e rendimento do estudante de e-learning.

Mais estudos são necessários para investigar de que modo o estudante de e-learning administra seu tempo e supera os desafios impostos pelo estudo em diferentes ambientes. Os estudos sobre evasão e persistência apresentados a seguir, indicam a importância de variáveis pessoais e contextuais na participação de alunos (adultos) em cursos a distância.

\section{Evasão em EAD: uma breve análise da literatura}

Um dos principais problemas da EAD e do TAD é a evasão de estudantes. Há casos em que apenas metade dos estudantes concluiu os treinamentos. De acordo com Amidani (2004), a evasão é um problema recorrente no ensino brasileiro e tem gerado muitas pesquisas com foco no ensino fundamental e médio na modalidade presencial. Na literatura internacional e nacional, encontram-se alguns estudos na área de EAD, no entanto, a maioria enfoca esse fenômeno relacionado ao ensino superior. Há também estudos que tratam da persistência de alunos em cursos a distância.

De acordo com Amidani (2004), um dos autores mais citados sobre evasão no ensino superior é Tinto (citado por Amidani, 2004). O autor propôs um modelo teórico para o estudo do fenômeno da permanência e da evasão em cursos de graduação. O modelo supõe que a persistência é função do compromisso do estudante para concluir o curso, do comprometimento do estudante com obrigações externas ao ambiente acadêmico, da formação escolar anterior, da integração acadêmica (intelectual) e da integração social do aluno (pessoal). As duas últimas são essenciais para a persistência e diferenças pessoais (demográficas) são menos importantes na determinação da evasão. Esse modelo tem sido aplicado para estudar evasão e persistência em cursos de graduação. Não se sabe, entretanto, se esse modelo é aplicável no contexto da evasão na educação corporativa, uma vez que há inúmeras diferenças entre os cursos de graduação e os treinamentos oferecidos pelas organizações aos seus empregados ou servidores.

No Brasil, o tema sobre evasão não figura entre os mais pesquisados no Brasil, segundo o ABRAEAD (Instituto Monitor, 2008). Para essa instituição, o índice de evasão dos cursos é variável nas empresas (Instituto Monitor, 2007). Os dados mostraram que 59,30\% das empresas registraram até $20 \%$ de evasão, enquanto que $18,50 \%$ registraram evasão de 20 a $30 \%$ e $22,20 \%$ das empresas registraram um índice preocupante maior do que $30 \%$.

Em 2008, a evasão continuou alta. De um total de $300 \mathrm{mil}$ participantes em todos os cursos oferecidos pela amostra de 41 organizações estudadas, 55\% foram indicados por empresas com evasão superior a $30 \%$. O número de participantes de empresas com menor índice de evasão (até $10 \%$ ) representa apenas $25 \%$ do total de participantes. As empresas pesquisadas citaram algumas desvantagens da EAD, entre as quais, estão: o custo de implantação, a impessoalidade, a ausência de intimidade com o método e a evasão.

Em 2008 o referido anuário relatou uma pesquisa junto a 102 estudantes evadidos de cursos a distância para identificar os fatores que pesaram na decisão de abandonar o curso e para descrever o padrão de ocorrência da evasão. Os resultados dessa pesquisa mostraram que a quase totalidade dos evadidos $(91,2 \%)$ abandonou o curso antes da metade do seu desenvolvimento, e $85 \%$ o abandonaram no início. Resultado similar a esse também foi encontrado por Almeida (2007).

Entre os motivos da evasão, a falta de tempo foi a causa mais citada com 53\% dos estudantes. Um segundo motivo, referiu-se à situação financeira, citado por $35 \%$ dos estudantes pesquisados. Os motivos restantes estão relacionados a não ter se adaptado ao sistema de curso a distância (15,6\%); a não ter se dedicado tanto quanto poderia ou deveria aos estudos (15,2\%); à escola não ter oferecido os recursos necessários (15,2\%); ao curso não ser exatamente o que queria $(10 \%)$; à localização da instituição $(8,2 \%)$; e à ausência de interação com outros estudantes $(6,1 \%)$. Entre os motivos, aparecem apenas três relacionados diretamente à modalidade de ensino ou qualidade das condições de ensino: falta de recursos de apoio; objetivos do curso pouco claros porque não eram compatíveis com expectativas do estudante e ausência de interação entre colegas.

A seguir é apresentada uma análise de uma amostra de pesquisas referentes à evasão, realizadas por pesquisadores brasileiros e estrangeiros, localizados a partir das bases de dados mencionadas anteriormente. A análise da amostra de artigos indica que os fatores associados à evasão podem ser classificados em duas grandes categorias: fatores ligados ao aluno e ao seu contexto, e fatores ligados ao desenho do e-learning e blended learning.

Entre os fatores ligados ao aluno e ao seu contexto, estão os seguintes: falta de tempo, dificuldades financeiras, falta de condições de estudo no local de trabalho, problemas relacionados ao trabalho, falta de condições de estudo em casa, razões pessoais ou domésticas, dificuldade para deslocar-se até o local destinado a encontros presenciais, falta de habilidade para administrar o tempo de estudo, falta de habilidade para conciliar as atividades concorrentes com as tarefas exigidas pelo curso, falta de experiência como aluno em cursos a distância, dificuldade para redigir textos e mensagens, falta de habilidade para utilizar recursos da internet, problemas com a tecnologia (falta de habilidade para realizar downloads de documentos e recursos de áudio e vídeo e para utilizar os recursos da internet), 
falta de suporte de outros alunos para atingir bons resultados de aprendizagem, não utilização de computador em outras tarefas, percepções equivocadas sobre a natureza e a complexidade do curso e baixa freqüência de participação do aluno em atividades de interação com o tutor e colegas (Almeida, 2007; Amidani, 2004; Brauer, 2005; Coelho Jr., 2004; Ngoma, Simwanza, \& Makunka, 2004; Pierrakeas, Xenos, Panagiotakopoulos, \& Vergidis, 2004; Walter, 2006; Yukselturk \& Inan, 2006).

Entre os fatores ligados ao desenho do e-learning e blended learning, estão: o não atendimento de expectativas pessoais; a falta de informações sobre a importância do curso; a baixa freqüência de uso das ferramentas da web; a insatisfação com o desempenho do tutor; o absenteísmo dos tutores; a falta de assistência do tutor ao aluno; o atraso no envio de feedbacks ou fornecimento de poucos informativos aos alunos; a separação física entre professor e alunos; a duração e a dificuldade do curso; o modo de entrega do curso (dificuldade em acessar o website); a quantidade de trabalhos escritos exigidos; o nível do curso; a falta de atividades face a face; a falta de suporte da unidade de ensino ao aluno; a má qualidade dos materiais de ensino e a falta de interação com colegas (Abbad, Carvalho, \& Zerbini, 2006; Almeida, 2007; Amidani, 2004; Coelho Jr., 2004; Pierrakeas et al., 2004; Walter, 2006).

A persistência em cursos a distância está associada a: conhecimento prévio sobre conteúdos semelhantes aos abordados pelo curso; motivação pessoal; necessidade e capacidade de balancear família e carreira; independência; autodisciplina (menor evasão); nível de escolaridade (quanto maior a escolaridade, menor a evasão) e natureza compulsória do curso implicando participação obrigatória do empregado ou servidor público no evento. Os resultados das pesquisas sobre a influência de idade e sexo sobre evasão em cursos a distância são inconsistentes e não conclusivos (Almeida, 2007; Amidani, 2004; Coelho Jr., 2004; Pierrakeas et al., 2004; Vargas, 2004; Walter, 2006; Wang, Foucar-Szocki, Griffin, O'Connor, \& Sceiford, 2003). A seguir, finalizando a análise dos artigos em função das categorias citadas anteriormente, apresenta-se a análise da produção de conhecimentos sobre avaliação de treinamentos e cursos a distância.

\section{Avaliação de treinamentos e cursos a distância}

Há poucos estudos sobre a eficácia dos métodos apoiados nos recursos da internet nos quatro níveis de avaliação de Kirkpatrick - reações, aprendizagem, comportamento no cargo, valor final (Burgess \& Russel, 2003).

Quanto às reações de participantes, professores e estudantes sobre cursos a distância mistos e puros, há resultados indicando um predomínio de percepções favoráveis a essa modalidade. Em ambientes universitários brasileiros, de acordo com o Instituto Monitor (2008), para a quase metade de alunos formados (46\%) a educação a distância é melhor do que a educação presencial, e para $33 \%$ é a mesma coisa. Apenas $16 \%$ a consideram pior. Na educação corporativa brasileira, o grau de adesão dos funcionários aos cursos a distância é excelente $(54,1 \%)$ ou bom $(35,1 \%)$. O grau de satisfação dos funcionários com os cursos a distância é bastante favorável, pois na maior parte das empresas (entre as 41 pesquisadas) a avaliação atingiu o conceito Bom
(54,1\%) ou Excelente (43,2\%). Nesses contextos de educação corporativa, o e-learning é a mídia mais utilizada $(97,1 \%)$ e o material impresso também é usado por uma maioria (65\%) das empresas. Entre as vantagens da EAD apontadas pelas empresas pesquisadas estão: a flexibilidade de tempo para o aluno $(94,4 \%)$, a agilidade (86,1\%), a abrangência e o alcance $(83,3 \%)$, a redução de cursos $(80,65)$ e o acesso facilitado para o aluno $(77,8 \%)$.

As avaliações favoráveis de cursos online puros e híbridos se repetem em outros contextos, inclusive nos ambientes universitários. Por exemplo, as reações favoráveis de alunos e professores ao blended learning em universidade estadunidense confirmam outros resultados referentes a cursos mediados pela web (Vaughan, 2007). O survey desse autor mostrou que: (a) $80 \%$ dos alunos de graduação da universidade pesquisada disse estar satisfeito com o curso híbrido; (b) o estudo em casa foi considerado mais agradável do que na universidade; (c) todos os professores gostaram da experiência e disseram que pretendiam repeti-la no futuro; (d) os gestores da universidade ressaltaram vários aspectos positivos da adoção do blended learning nos cursos, entre eles a melhoria da reputação da instituição, a expansão do acesso de alunos aos cursos oferecidos pela instituição, e a redução dos custos de operação.

De Paula e Silva (2004) relatou reações favoráveis de alunos de graduação da Universidade de Brasília a uma disciplina oferecida no formato misto. Os alunos avaliaram positivamente os aspectos ergonômicos do ambiente virtual do curso, os procedimentos instrucionais e o serviço de tutoria. Nogueira (2006) também relatou avaliações favoráveis de um treinamento auto-instrucional mediado pela internet sobre técnicas de secretariado. $\mathrm{O}$ treinamento foi positivamente avaliado em diversos aspectos, entre os quais: a qualidade da interface gráfica do ambiente virtual de aprendizagem, os resultados ao final do curso e as situações de ensino-aprendizagem oferecidas ao participante.

Quanto à efetividade de treinamentos à distância, mediados por recursos da $w e b$, no nível de aprendizagem ou de rendimento dos participantes, observam-se resultados igualmente favoráveis para a modalidade. Uma das maneiras de investigar essa questão investigada tem sido a comparação entre as modalidades a distância e com presença, de modo a verificar se a aprendizagem no e-learning e no blended learning é igual ou superior à obtida em cursos tradicionais com presença. Quanto ao grau de aprendizagem, não há dados conclusivos, mas os predominantes indicam que a aprendizagem a distância é igual ou superior à atingida por estudantes em cursos tradicionais.

Há poucas diferenças quanto ao formato das avaliações de aprendizagem em e-learning e cursos tradicionais. A revisão de Burgess e Russel (2003), apoiada em revisões anteriores sobre avaliação de aprendizagem a distância em organizações, localizou poucos estudos sobre a eficácia de métodos de aprendizagem a distância para promover a aprendizagem no trabalho. Os autores fazem referência a uma revisão sobre efetividade de aprendizagem a distância, que abrangeu 250 estudos em um período de trinta anos e observou que não havia diferenças significativas no rendimento de estudantes de cursos a distância e de classes tradicionais. 
Em um dos estudos analisados por Burgess e Russel (2003), foi observado que os alunos de classes tradicionais obtiveram notas mais altas do que os de aprendizagem a distância em uma indústria nos EUA. Em organizações do governo federal norteamericano, observou-se que os participantes de cursos a distância obtiveram melhores notas do que os que participaram de cursos tradicionais de sala de aula.

No ABRAED (Instituto Monitor, 2008), Litto afirma que, segundo os resultados do Exame Nacional de Desempenho de Estudantes (ENADE) de 2006, aplicado nos egressos de cursos superiores, em sete dos treze domínios de conhecimento acadêmico, os alunos que estudaram a distância obtiveram notas melhores em relação àqueles que estudaram em cursos presenciais e que, em todas as áreas, os estudantes de cursos a distância obtiveram notas ligeiramente maiores do que as de egressos de cursos com presença.

Entre os sete cursos a distância cujos egressos obtiveram melhores notas no ENADE 2006, estão: Administração, Biologia, Ciências Sociais, Física, Matemática, Pedagogia e Turismo. Para o autor, não é novidade que a EAD é eficaz e que, em alguns casos, a educação a distância é superior à modalidade presencial no ensino universitário. Isto se deve, na visão do autor, a dois fatores: (a) o aluno que escolhe o curso a distância é psicologica e cronologicamente mais maduro, tal como demonstram as estatísticas do ABREAD (Instituto Monitor, 2008) e, consequentemente, mais motivado e disciplinado; (b) enquanto o ensino presencial depende fortemente do professor, que trabalha de modo independente e isolado, a EAD é sempre feita por uma equipe de especialistas, que se preocupa com a qualidade dos materiais e condições de aprendizagem em diversos aspectos. A EAD depende dos materiais e de outras condições de ensino-aprendizagem, que, geralmente precisam ser confeccionadas após um cuidadoso planejamento e com a preocupação de incorporar algumas funções didáticas do bom professor.

Entre os artigos analisados nesta revisão, foram encontradas relativamente poucas pesquisas sobre comportamento no cargo (transferência de treinamento, impacto do treinamento no trabalho em profundidade e impacto em amplitude), entre as quais há estudos realizados no Brasil (Brauer, 2005; Carvalho, 2003; Coelho Jr., 2004; Zerbini, 2003, 2007).

A seguir, apresenta-se um resumo dos aspectos metodológicos e resultados de uma amostra não exaustiva de pesquisas brasileiras que descrevem variáveis explicativas de reações, aprendizagem e impacto do treinamento no trabalho.

Das oito pesquisas brasileiras analisadas (Borges-Ferreira, 2005; Carvalho, 2003; Coelho Jr., 2004; De Paula e Silva, 2004; Nogueira, 2006; Vargas, 2002; Zerbini 2003; Zerbini, 2007), apenas três foram realizadas em empresas (Coelho Jr., 2004; Nogueira, 2006; Vargas, 2002). As demais foram conduzidas em contextos de formação e qualificação profissional (BorgesFerreira, 2005; Carvalho, 2003; Zerbini 2003, 2007) e uma em ambiente universitário (De Paula e Silva, 2004).

Seis pesquisas avaliaram reações (Borges-Ferreira, 2005; Carvalho, 2003; De Paula e Silva, 2004; Vargas, 2002; Zerbini 2003, 2007), quatro avaliaram aprendizagem (Borges-Ferreira,
2005; Carvalho, 2003; Coelho Jr., 2004; Nogueira, 2006) e cinco avaliaram impacto do treinamento no trabalho (Carvalho, 2003; Coelho Jr., 2004; Nogueira, 2006; Zerbini 2003, 2007). Metade dos artigos avaliou somente um desses resultados. Nesta amostra de artigos não foram localizadas avaliações do impacto de treinamentos a distância nos níveis de mudança organizacional e valor final.

Os resultados das investigações mostraram que variáveis pessoais dos estudantes como estratégias de aprendizagem, objetivos extrínsecos, valor instrumental, experiência de participação em cursos a distância e habilidades de utilizar a internet, em especial as ferramentas de interação e comunicação, estão positivamente relacionadas a reações e, nas pesquisas anteriormente analisadas, estão negativamente relacionadas com evasão em cursos a distância.

Reações favoráveis dos estudantes ao treinamento (procedimentos de ensino-aprendizagem, materiais, resultados e aplicabilidade e à interface gráfica do ambiente virtual de aprendizagem) estão relacionadas com transferência de treinamento. Porém, estão fracamente relacionadas com aprendizagem, corroborando resultados obtidos em avaliações de treinamentos presenciais.

Variáveis relativas ao desenho instrucional de situações de aprendizagem tal como a qualidade da interação entre tutores e aprendizes, qualidade do conteúdo e dos feedbacks, e tempo de exposição dos feedbacks ao aluno, estão associados à persistência e à aprendizagem em situações de e-learning e em blended learning.

Há resultados indicando que, além de variáveis do contexto de trabalho, variáveis pessoais relacionadas à família do estudante também afetam a evasão e a aprendizagem ou o rendimento do estudante em cursos a distância. Os resultados das investigações mostram que variáveis pessoais dos estudantes como a idade, locus de controle (Parker, 1995), estratégias de aprendizagem, hábitos de estudo, motivação para aprender, valor instrumental, experiência de participação em cursos a distância e habilidades de utilizar a internet estão relacionadas a reações e níveis de evasão em cursos a distância.

A análise mostrou também um volume relativamente pequeno de estudos sobre transferência de treinamento, relevante fenômeno para a área de psicologia organizacional e do trabalho. A efetividade de cursos a distância em universidades não tem sido avaliada no nível de transferência. As pesquisas, em geral, lidam com avaliação de reações, aprendizagem e índices de evasão, mas não investigam o efeito exercido pelo e-learning sobre o desempenho posterior do egresso no ambiente de trabalho. Há poucos estudos sobre o impacto do e-learning ou blended learning sobre o desempenho posterior do egresso no trabalho. Entre essas pesquisas estão os estudos brasileiros de Carvalho (2003), Coelho Jr. (2004) e Zerbini (2003, 2007). Não foram localizados estudos comparando e-learning com aprendizagem espontânea nas organizações.

Não foram encontrados estudos sobre o impacto de treinamentos online sobre resultados organizacionais (mudança organizacional e valor final). O impacto de treinamentos sobre o desempenho da organização é também pouco estudado em 
contextos de treinamentos presenciais. Esses efeitos póstreinamento são relevantes e precisam ser estudados por meio de modelos de avaliação.

\section{Considerações finais}

A revisão da produção de conhecimentos em EAD no Brasil mostra claramente a necessidade de mais pesquisas nas áreas de educação corporativa, qualificação profissional e avaliação em EAD. Tal fato pode ser reflexo da trajetória relativamente recente de pesquisas brasileiras sobre o tema. No Brasil o uso do e-learning é crescente na educação corporativa e em universidades. Os dados do Instituto Monitor (2008) mostraram um panorama da EAD em instituições de ensino e na educação corporativa no Brasil. Mais de dois milhões e meio de brasileiros estudaram por meio de educação a distância em 2007, na forma de cursos credenciados e oferecidos oficialmente, de grandes projetos nacionais públicos e privados e de educação corporativa. É crescente o número de instituições de ensino autorizadas pelo governo a oferecer cursos a distância. O número de alunos que estuda em instituições de ensino superior credenciadas cresceu muito, passando de 309.957 alunos (em 2004) para 972.826 (em 2007). Na educação corporativa brasileira, os dados sugerem que as 41 organizações abordadas pela pesquisa investiram mais de um quarto $(25,6 \%)$ dos recursos em EAD.

A análise dos artigos mostrou que a produção de conhecimentos sobre a eficácia do e-learning e do blended learning, em ambientes organizacionais, ainda é incipiente. O ritmo das pesquisas é incompatível com o acelerado crescimento desse tipo de aprendizagem em organizações. Além disto, a produção é dispersa e fragmentada, tornando difícil a comparação entre os resultados dos estudos e lento o avanço do conhecimento sobre aprendizagem a distância em organizações.

Grande parte da amostra de artigos relata estudos realizados em cursos a distância, oferecidos por universidades, e foi publicada em revistas das áreas de educação e ciência da computação. Foram encontrados poucos artigos publicados em revistas da área de psicologia. Observou-se produção de conhecimentos veiculada em revistas de nutrição, medicina e ergonomia, o que indica a natureza multifacetada e multidisciplinar dos assuntos concernentes à EAD e à TAD.

Segundo pesquisadores da área, tais como Abbad, Carvalho e Zerbini (2006), Burgess e Russell (2003), Sonnentag, Niessen e Ohly (2004), entre outros, há evidências de que suporte à transferência exerce influência positiva sobre a aplicação de novas aprendizagens no trabalho. Entretanto, poucos estudos investigam esse tipo de relacionamento entre variáveis. O suporte organizacional a empregados que participam de cursos online não é a única variável que influencia resultados de treinamentos a distância. Outras variáveis relacionadas aos diversos ambientes de estudo do estudante afetam os resultados de treinamentos a distância, mas tem sido pouco estudados.

Em estudos sobre transferência de treinamentos, realizadas por Carvalho (2003) e Carvalho e Abbad (2006), ficou evidente que a aplicação de novas aprendizagens no trabalho, em algumas situações, depende de suporte externo à organização. Na pesquisa de Carvalho (2003), a aplicação das habilidades de elaboração de um plano de negócios, ensinada gratuitamente a empreendedores pelo SEBRAE, dependia de múltiplas variáveis do macro ambiente, como: a qualidade das informações governamentais sobre o setor de negócios; a situação econômica do local; e as condições de infraestrutura e de segurança pública do local, onde o egresso do curso pretendia iniciar ou aprimorar seu empreendimento.

A aprendizagem oportunizada por cursos a distância, em especial os treinamentos online, aumenta as chances de participação desses adultos em treinamentos até o final do curso. O estudo assíncrono e veiculado por múltiplas mídias possibilita o acesso de profissionais à aprendizagem a qualquer hora e em qualquer lugar. Essa característica da modalidade a torna um importante meio de democratização e ampliação do acesso de minorias à aprendizagem e à qualificação profissional.

No que concerne ao desenho instrucional, a análise da literatura revela uma escassez de estudos sistemáticos sobre importantes questões ligadas, por exemplo, à influência do uso de organizadores gráficos e mapas conceituais sobre a aprendizagem, retenção, armazenagem, recuperação de informações na memória e na aplicação de novas habilidades no trabalho, e à influência de diferentes combinações de meios de ensino sobre a memorização, a aprendizagem e a transferência de aprendizagem.

Sugere-se como agenda de pesquisa: identificar variáveis de suporte dos contextos de estudo dos participantes de treinamentos online que interferem em sua aprendizagem, na motivação para aprender, na persistência no curso e na aplicação no trabalho das aprendizagens adquiridas no treinamento online; analisar a influência do desenho instrucional na memorização, na aprendizagem e na transferência de aprendizagem; e, finalmente, mas não menos importante, validar e aprimorar escalas de avaliação do efeito do e-learning sobre a aprendizagem e a transferência de aprendizagem para o trabalho.

Por fim, vale resgatar que a análise aqui efetuada, ao referendar-se em elementos e implicações teóricas, empíricas e metodológicas da situação atual de estudos em EAD, lança novas luzes para pesquisas futuras sobre a aprendizagem no trabalho e nas organizações.

\section{Referências}

Abbad, G. S. (2007). Educação a distância: o estado da arte e o futuro necessário. Revista do Serviço Público (Brasília), 58, 100-110.

Abbad, G. S., Carvalho, R. S., \& Zerbini, T. (2006). Evasão em curso via internet: explorando variáveis explicativas. Revista de Administração de Empresas Eletrônica, 5(2). Recuperado de http://www.scielo.br/pdf/raeel/ v5n2/v5n2a08.pdf

Almeida, O. C. S. (2007). Evasão de cursos a distância: validação de instrumento, fatores influenciadores e cronologia da desistência (Dissertação de mestrado, Universidade de Brasília, Brasília).

Amidani, C. (2004). Evasão no ensino superior a distância: o curso de licenciatura em matemática a distância da Universidade Federal Fluminense/CEDERJ7 - RJ (Dissertação de mestrado, Universidade de Brasília, Brasília).

Borges-Ferreira, M. F. (2005). Avaliação de reações e aprendizagem em disciplinas de curso técnico profissionalizante oferecidas a distância (Dissertação de mestrado, Universidade de Brasília, Brasília).

Brauer, S. (2005). Avaliação de um curso a distância: valor instrumental do 
treinamento, barreiras pessoais à conclusão e evasão (Dissertação de mestrado, Universidade de Brasília, Brasília).

Burgess, J. R. D., \& Russell, J. E. A. (2003). The effectiveness of distance learning iniciatives in organizations. Journal of Vocational Behavior, 63, 289-303.

Carvalho, R. S. (2003). Avaliação de treinamento a distância via internet: reação, suporte à transferência e impacto do treinamento no trabalho (Dissertação de mestrado, Universidade de Brasília, Brasília).

Carvalho, R. S., \& Abbad, G. S. (2006). Avaliação de treinamento a distância: reação, suporte à transferência e impactos no trabalho. Revista de Administração Contemporânea, 10(1), 95-116.

Coelho Jr., F. A. (2004). Avaliação de treinamento a distância: suporte à aprendizagem e impacto do treinamento no trabalho (Dissertação de mestrado, Universidade de Brasília, Brasília).

Coelho Jr., F. A., Abbad, G. S., \& Vasconcelos, L. C. (2008). Análise da relação de clientela, suporte à aprendizagem e impacto de treinamento a distância. Revista de Administração Contemporânea, 22(1), 88-104.

Coelho Jr., F. A., \& Borges-Andrade, J. E. (2008). Uso do conceito de aprendizagem em estudos relacionados ao trabalho e organizações. Paidéia, 18(40), 221-234.

De Paula e Silva, A. (2004). Avaliação de uma disciplina semipresencial de graduação ofertada por meio da internet pela Universidade de Brasília (Dissertação de mestrado, Universidade de Brasília, Brasília).

Gormley, G., Collins, K., Boohan, M., Bickle, I., \& Stevenson, M. (2009). Is there a place for e-learning in clinical skills? A survey of undergraduate medical students' experiences and attitudes. Medical Teacher, 31(1), e6-e12.

Instituto Monitor (2007). Anuário Brasileiro Estatístico de Educação Aberta e a Distância. São Paulo: Autor.

Instituto Monitor (2008). Anuário Brasileiro Estatístico de Educação Aberta e a Distância. São Paulo: Autor.

Kelly, M., Lyng, C., McGrath, M., \& Cannon, G. (2009). A multi-method study to determine the effectiveness of, and student attitudes to, online instructional videos for teaching clinical nursing skills. Nurse Education Today, 29(3), 292-300.

Ngoma, P. S., Simwanza, A., \& Makunka, C. K. (2004). Investigating the drop out problem amongst university extensión studies learners in Zambia. Recuperado de http://www.col.org/pcf3/Papers/PDFs/Ngoma_Simwanza_ Makunka.pdf

Nogueira, R. S. F. (2006). Efeitos do tempo de exposição ao feedback na aprendizagem em treinamento baseado na web (Dissertação de mestrado, Universidade de Brasília, Brasília).

Parker, A. (1995). Distance education attrition. International Journal of
Educational Telecommunications, 1(4), 389-406.

Parker, A. (1999). A study of variables that predict dropout from distance education. International Journal of Educational Technology, 1(2), 1-10.

Pierrakeas, C., Xenos, M., Panagiotakopoulos, C., \& Vergidis, D. (2004). A comparative study of dropout rates and causes for two different distance education courses. The International Review of Research in Open and Distance Learning, 5(2), 1-15.

Shemla, A., \& Nachmias, R. (2007). Current state of web-supported courses at Tel-Aviv University. International Journal on E-learning, 6(2), 235-246.

Sonnentag, S., Niessen, C., \& Ohly, S. (2004). Learning at work: training and development. In C. L. Cooper \& I. T. Robertson(Orgs.), International Review of Industrial and Organizational Psychology: vol. 19 (pp. 249-289). New York: John Wiley \& Sons Ltda.

Vargas, M. R. M. (2002). Educação a distância e as novas tecnologias: o uso da videoconferência em treinamentos organizacionais. Revista Brasileira de Aprendizagem Aberta e a Distância, 1. Recuperado de http://www.abed.org. br/revistacientifica/_brazilian/edicoes/2002/2002_Edicao.htm

Vargas, M. R. M. (2004). Barreiras à implantação de programas de educação e treinamento a distância (Tese de doutorado, Universidade de Brasília, Brasília).

Vaughan, N. (2007). Perspectives on blended learning in higher education. International Journal on E-learning, 6(1), 81-94.

Walter, A. M. (2006). Variáveis preditoras de evasão em cursos a distância (Dissertação de mestrado, Universidade de Brasília, Brasília).

Wang, G., Foucar-Szocki, D., Griffen, O., O'Connor, C., \& Sceiford, E. (2003). Departure, abandonment, and dropout of E-learning: dilemma and solutions. Recuperado de http://www.titaylor.net/research/DepartureAbandonment-Dropout-of-Elearning-Dilemma-and-Solutions-JamesMadison-University-2003.pdf

Xenos, M., Pierrakeas, C., \& Pintelas, P. (2002). A survey on student dropout rates and dropout causes concerning the students in the course of informatics of the Hellenic Open University. Computers \& Education, 39, 361-377.

Yukselturk, E., \& Inan, F. A. (2006). Examining the factors affecting student dropout in an online learning environment. Turkish Online Journal of Distance Education, 7(2), 76-88.

Zerbini, T. (2003). Estratégias de aprendizagem, reações aos procedimentos de um curso via internet, reações ao tutor e impacto do treinamento no trabalho (Dissertação de mestrado, Universidade de Brasília, Brasília).

Zerbini, T. (2007). Avaliação da transferência de treinamento em curso a distância (Tese de doutorado, Universidade de Brasília, Brasília).

Gardênia da Silva Abbad, doutora em Psicologia pela Universidade de Brasília, é Diretora do Instituto de Psicologia da Universidade de Brasília. Endereço para correspondência: Campus Universitário - Instituto de Psicologia-UnB, Asa Norte, CEP.: 70910-900, Brasília-DF.Tel.: (61) 3307-2625 Ramal: 222. E-mail: gardenia.abbad@gmail.com

Thaís Zerbini, doutora em Psicologia pela Universidade de Brasília, é Vice-coordenadora do curso de Psicologia da Universidade de São Paulo, Ribeirão Preto. E-mail: thais.zerbini@gmail.com Daniela Borges Lima de Souza, mestre em Psicologia Social pela Universidade Gama Filho, é doutoranda em Psicologia pela Universidade de Brasília. E-mail: danisssol@yahoo.com 